\title{
Interspecific ICSI for the Assessment of Sperm DNA Damage: Technology Report
}

\author{
Jana Rychtarova ${ }^{1, *}$, Alena Langerova ${ }^{2}$, Helena Fulka ${ }^{1,3}\left(\mathbb{D}\right.$, Pasqualino Loi ${ }^{4}\left(\mathbb{D}\right.$, Michal Benc ${ }^{1,5}(\mathbb{D}$ \\ and Josef Fulka Jr. ${ }^{1}$ \\ 1 Institute of Animal Science, 10400 Prague, Czech Republic; helena.fulka@gmail.com (H.F.); \\ benc.michal@gmail.com (M.B.); fulka.josef@vuzv.cz (J.F.J.) \\ 2 GENNET, 17000 Prague, Czech Republic; alena.langerova@gennet.cz \\ Institute of Experimental Medicine, 14220 Prague, Czech Republic \\ 4 Faculty of Veterinary Medicine, University of Teramo, 64100 Teramo, Italy; valutatorearea07@unite.it \\ 5 Faculty of Natural Sciences, Constantine the Philosopher University in Nitra, 94974 Nitra, Slovakia \\ * Correspondence: rychtarova.jana@vuzv.cz; Tel.: +420-267009801
}

check for updates

Citation: Rychtarova, J.; Langerova, A.; Fulka, H.; Loi, P.; Benc, M.; Fulka, J., Jr. Interspecific ICSI for the Assessment of Sperm DNA Damage: Technology Report. Animals 2021, 11, 1250. https://doi.org/10.3390/ ani11051250

Academic Editor: Emilia Bagnicka

Received: 25 March 2021

Accepted: 23 April 2021

Published: 26 April 2021

Publisher's Note: MDPI stays neutral with regard to jurisdictional claims in published maps and institutional affiliations.

Copyright: (c) 2021 by the authors. Licensee MDPI, Basel, Switzerland. This article is an open access article distributed under the terms and conditions of the Creative Commons Attribution (CC BY) license (https:// creativecommons.org/licenses/by/ $4.0 /)$.
Simple Summary: The long-term storage of biological material (sperm, oocytes, embryos, etc.) is essential not only for animal breeding programs, human ART, and basic biology, but also for rescuing endangered animal species and other technologies. Whilst sperm storage is almost perfected for some species (bovine, human, mouse), many problems remain in others. In our contribution, we present a simple approach that can be used for the rapid evaluation of DNA damage level in sperm nuclei after freezing. This approach can be useful especially in those cases when the amount of frozen biological material is limited and permits much higher flexibility when modifying chosen preservation approaches.

Abstract: Xenogenic mammalian sperm heads injected into mouse ovulated oocytes decondense and form pronuclei in which sperm DNA parameters can be evaluated. We suggest that this approach can be used for the assessment of sperm DNA damage level and the evaluation of how certain sperm treatments (freezing, lyophilization, etc.) influence the quality of spermatozoa.

Keywords: sperm; oocyte; DNA damage

\section{Introduction}

Mammalian spermatozoa only exceptionally fertilize intact or zona-free oocytes of another mammalian species. The only exception seems to be the combination of hamster zona-free oocytes, into which mammalian spermatozoa of other species penetrate quite frequently when in vitro fertilization (IVF) is used. After the penetration, the sperm heads decondense in a foreign cytoplasm and form pronuclei. Nevertheless, in this case the spermatozoa must be highly motile [1,2]. High motility, however, does not mean that the sperm DNA is not damaged. At the same time, it also does not mean that nonmotile sperm cannot produce functional pronuclei. Indeed, it has been demonstrated that immotile and even dead spermatozoa, for example, when lyophilized or recovered from frozen cadavers, can produce normal offspring when injected into the same species oocyte $[3,4]$. On the other hand, the motile spermatozoa with damaged DNA (i.e., after chemotherapy) can fertilize the oocyte but subsequent development is compromised [5]. Typically, these spermatozoa decondense in the cytoplasm and form pronuclei with delayed DNA replication; further cleavage is abnormal, and micronuclei can be detected in the cytoplasm of the two-cell embryos [6,7].

Many factors can damage sperm DNA. This can occur already during spermiogenesis by excessive ROS (reactive oxygen species) generation, by anticancer drug treatments, smoking, air pollution, etc. [8]. Beside this, sperm DNA can be damaged even when 
conventionally used techniques are inappropriately applied, e.g., ICSI (intracytoplasmic sperm injection) [9] or during the sperm heads preparation for ICSI [10,11]. Specific attention must also be paid to some sperm storage procedures such as cryopreservation and lyophilization [12].

There are many approaches that can be used for the evaluation of sperm DNA damage. Typically, these approaches need a relatively high number of spermatozoa for evaluation; therefore, they cannot be used, for example, for oligospermic samples [13].

Assessing the intensity of $\gamma \mathrm{H} 2 \mathrm{AX}$ labeling is a commonly used approach for the evaluation of DNA damage in somatic cell nuclei. Histone $\mathrm{H} 2 \mathrm{AX}$ is rapidly phosphorylated at sites of DNA double-strand breaks (DSBs), and this phosphorylated H2AX $(\gamma \mathrm{H} 2 \mathrm{AX})$ then recruits numerous repair proteins [14-16].

Our "technology report" presents a relatively simple approach that can be used for sperm DNA damage evaluation, especially in those cases where sperm numbers are very low.

\section{Materials and Methods}

Mouse oocytes were isolated from oviducts of superovulated B6D2F1 females (PMSG 5 IU i.p. with hCG 5 IU i.p. approximately 44 h post PMSG; Intervet, Boxmeer, The Netherlands). The ovulated oocytes were isolated from oviduct ampullae after about $14-15 \mathrm{~h}$ post hCG and incubated in M2 medium supplemented with hyaluronidase $(0.1 \%)$. This incubation facilitates the removal of cumulus cells by vigorous pipetting. The oocytes were then cultured briefly in KSOM (Millipore, Prague, Czech Republic) at $37^{\circ} \mathrm{C}$ in a humified incubator $\left(5 \% \mathrm{CO}_{2}\right.$ in air) and then used for intracytoplasmic sperm injection (ICSI).

For ICSI, we used fresh or cryopreserved goat, ram, bovine, pig, horse and rooster spermatozoa from Accredited Insemination Stations. The frozen samples were thawed in a water bath $\left(37^{\circ} \mathrm{C}\right)$, washed several times with $\mathrm{M} 2$ medium and then used for ICSI. As this is a methodological paper, for ICSI we used two extremes-fresh (highly vital — motile with sharp contours) or badly frozen, i.e., mostly immotile ( $10 \%$ motile)/no sharp contours sperm samples.

ICSI was performed essentially as described by Yoshida and Perry [17]. Briefly, the oocytes (10-20) and spermatozoa were placed into $10 \mu \mathrm{L}$ of M2 covered with paraffin oil on the lid of a $10 \mathrm{~cm}$ Petri dish. Isolated sperm heads (whole rooster sperm) were injected into oocytes with a piezo injector PMAS-CT150 (Tsukuba, Ibaraki, Japan) on the inverted microscope Olympus stage IX 71 (Olympus, Prague, Czech Republic), magnification $40 \times$.

Immediately after the sperm head injection, the oocytes (zygotes) were washed several times in KSOM and cultured in it for $\sim 9 \mathrm{~h}$ as described above. Then, the interspecific zygotes were inspected under the inverted Olympus IX 71 microscope with Hoffman optics, selected, and suitable oocytes transferred into M2 - their zonae pellucidae were removed by acid Tyrode solution and zona-free zygotes were fixed in $4 \%$ paraformaldehyde for $15 \mathrm{~min}$ at room temperature. The fixed samples were then kept in PBS (Phosphate Buffered Saline) in a refrigerator before labeling.

The fixed samples were first permeabilized in 0.2\% Triton X-100 (Sigma, Prague, Czech Republic) in PBS for 10 min at room temperature (RT) and then blocked overnight in 1\% BSA,(Bovine Serum Albumin) 0.1\% TX-100 in PBS in a refrigerator. The samples were then incubated in the same solution with the first antibody for $1 \mathrm{~h}$ : $\gamma \mathrm{H} 2 \mathrm{AX} 1: 200$ (Abcam, Cambridge, UK), and then, after extensive washing, in PBS/BSA in the appropriate secondary antibody (Alexa Fluor donkey anti rabbit, 1:800, Jackson ImmunoResearch, Ely, UK) for $2 \mathrm{~h}$. After extensive washing, the samples were mounted in Vectashield and evaluated under the fluorescence microscope Olympus BX 61 (Olympus, Prague, Czech Republic).

Each ICSI combination was repeated at least three times with more than 50 oocytes injected for every species.

If not stated otherwise, all chemicals were purchased from Sigma, Prague, Czech Republic. 


\section{Results}

The survival of injected oocytes was almost absolute and only exceptionally they died during or immediately after injection. More than $90 \%$ of oocytes were activated when injected with the sperm, i.e., they extruded the second polar body and contained at least the maternal (female) pronucleus (fPN). With the exception when rooster spermatozoa were used (see below), in about $75 \%$ of activated oocytes two PNs were visible already under the stereomicroscope (summarized in Table 1). No intact sperm heads were detected in activated oocytes with a single pronucleus, when stained with Hoechst. These oocytes were discarded and not used for further labeling.

Table 1. Xenogenic sperm head injection into mouse ovulated oocytes.

\begin{tabular}{ccccc}
\hline Sperm Origin & $\begin{array}{c}\text { No. of Oocytes } \\
\text { Injected/Survived (\%) }\end{array}$ & $\begin{array}{c}\text { Activated with Both } \\
\text { PNs and 2PB * }\end{array}$ & $\begin{array}{c}\gamma \text { H2AX in mPNs } \\
\text { (Fresh Sperm) } * *\end{array}$ & $\begin{array}{c}\gamma \text { H2AX in mPNs } \\
\text { (Frozen Sperm) }\end{array}$ \\
\hline horse & $52 / 49(94 \%)$ & $37(76 \%)$ & $15(+/-)$ & $20(+++)$ \\
goat & $67 / 62(93 \%)$ & $48(77 \%)$ & $20(+/-)$ & $24(+++)$ \\
bull & $51 / 49(96 \%)$ & $37(76 \%)$ & $17(+/-)$ & $18(+++)$ \\
ram & $63 / 59(94 \%)$ & $45(76 \%)$ & $20(+/-)$ & $22(+++)$ \\
pig & $60 / 58(97 \%)$ & $45(78 \%)$ & $22(+/-)$ & $21(+++)$ \\
Rooster $^{+}$ & $50 / 49(49 \%)$ & 0 & n.a. & n.a. \\
\hline
\end{tabular}

* The remaining oocytes extruded the second polar body and contained only a single PN. No intact sperm heads were detected in the oocyte cytoplasm after Hoechst staining. ${ }^{* *}$ Some injected oocytes were lost during labeling. ${ }^{+}$Whole rooster sperm was injected into the mouse oocyte cytoplasm. +/ - Only minor labeling was detected over the mPNs with essentially no labeling in fPNs. +++ Heavy labeling over mPNs and no labeling in fPNs. n.a. Not applicable.

First, we wanted to know the response of different species sperm heads to the mouse oocyte cytoplasmic environment. The responses are graphically depicted in Figure 1 . When goat, ram, bovine and horse spermatozoa were injected into mouse oocytes, their sperm heads decondensed and formed paternal (male) pronuclei ( $\mathrm{mPNs}$ ) that were always larger than maternal (mouse) pronuclei (fPNs). The second polar body was extruded in all cases. fPNs were located in the vicinity of second polar bodies, whilst the mPNs were located more distantly. Paternal and maternal PNs contained distinct nucleolus precursor bodies (NPBs). Interestingly, pig sperm also activated the oocytes with the formation of normal fPNs, but the paternal PN was always slightly smaller. A more interesting situation was the combination: rooster $x$ mouse. Here, the sperm did not form PNs and only slightly decondensed, whilst fPNs were always present. What is also interesting is that highly motile rooster spermatozoa swam very actively in the mouse cytoplasm for a few minutes post injection.

After labeling against $\gamma \mathrm{H} 2 \mathrm{AX}$, only a minor signal in mPNs was observed when fresh sperm samples were used, and essentially no labeling in fPNs was observed-this was also observed for the pig. On the other hand, very intensive labeling in mPNs (sperm) was observed when improperly frozen samples were used for injection (Figure 2). No labeling was observed in almost unchanged rooster sperm heads and evidently these sperm heads did not respond to the mouse oocyte cytoplasm. Here, we labeled several oocytes with a lamin B antibody (not described in detail), and as evident from Figure 2, the nuclear lamina was present only in fPNs. However, we cannot rule out that the antibody does not recognize avian lamin $B$.

In conclusion, we demonstrate that interspecific ICSI can be used for the evaluation of sperm DNA damage. For practical purposes, it is, however, necessary to set the limits of damaged DNA which cannot be repaired by the oocyte. 


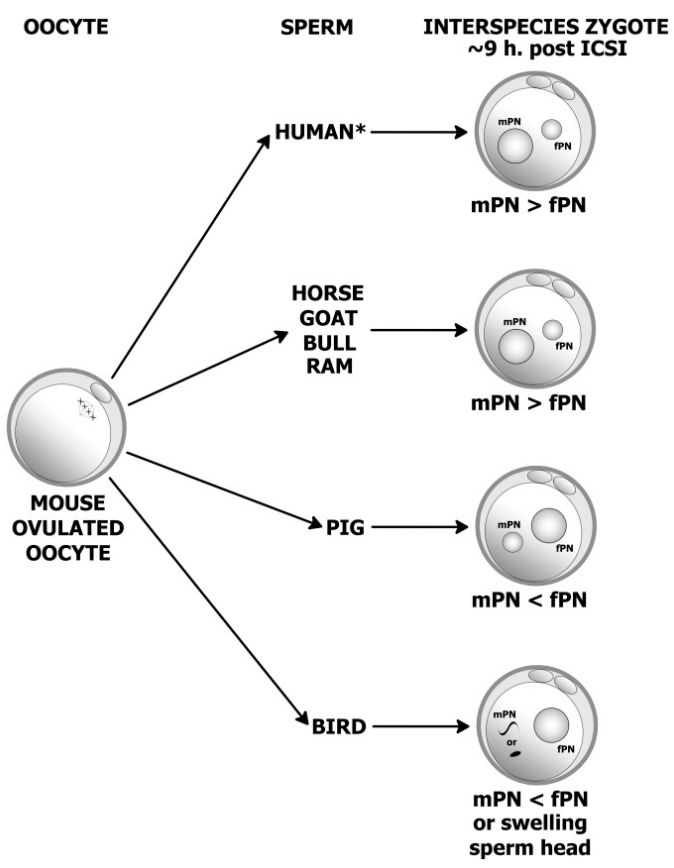

Figure 1. Decondensation of foreign sperm heads in ovulated mouse oocytes. Paternal pronuclei (mPNs) were always larger than maternal (female fPNs) when human, bull, horse, goat and ram spermatozoa were injected into ovulated mouse oocytes (* previous work: Fulka et al. [18]) and samples were evaluated approximately $9 \mathrm{~h}$ post ICSI. On the other hand, in the pig the mPNs were smaller than fPNs. When rooster spermatozoa were used for ICSI, no sperm head decondensation occurred (eventually only minor sperm head swelling was observed).
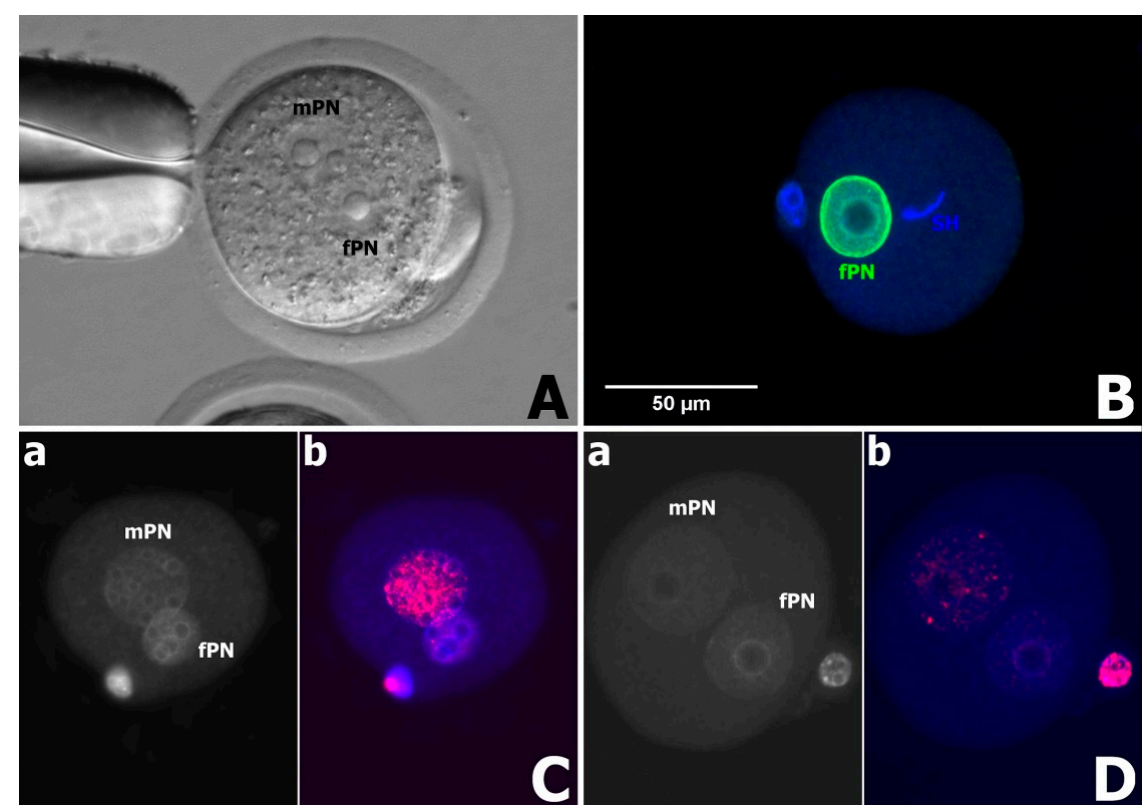

Figure 2. Representative pictures demonstrating the behavior of sperm heads after interspecific ICSI. (A) Goat sperm in mouse oocytes forms large $\mathrm{mPN}$ with several nucleoli. Maternal, female-fPN is always smaller. (B) Rooster sperm (SH) does not respond to mouse oocyte cytoplasm whilst the maternal (fPN) is normally formed. (C) Heavily damaged sperm DNA (mPN) exhibits very intensive labeling against $\mathrm{\gamma} H 2 \mathrm{AX}$ - (a) DNA staining with Hoechst (mPN—sperm, $\mathrm{fPN}$-maternal pronucleus), (b) parallel picture showing very intensive ${ }_{\gamma} \mathrm{H} 2 \mathrm{AX}$ signal in $\mathrm{mPN}$. (D) Only weak labeling against $\mathrm{\gamma H} 2 \mathrm{AX}$ is detected over mPNs when fresh spermatozoa are used for ICSI ((a)-Hoechst staining, (b) $-\mathrm{rH} 2 \mathrm{AX}$ labeling). 


\section{Discussion}

There are several approaches that can be used for the evaluation of sperm quality. Classically, sperm motility, viability, concentration and gross morphology serve as the basic criteria to evaluate the quality of semen. With the advent of more sophisticated approaches in ART (ICSI, ROSI, etc.) that can overcome immotility and low sperm numbers, more specific methods are being used (for example, the evaluation of acrosome morphology, sperm membrane intactness, etc.). Among them is the evaluation of DNA integrity, i.e., sperm DNA damage. Several approaches can be used for the assessment of DNA integrity-acridine orange test, sperm chromatin structure assay, chronomycin A3, aniline and toluide blue staining, in situ nick translation, terminal deoxy nucleotidyl transferase mediated dUTP nick end labeling assay (TUNEL), sperm chromatin dispersion, single cell gel electrophoresis or comet assay, etc. [19]. These approaches typically need a relatively high number of spermatozoa and show relatively low sensitivity.

In general, heavy sperm DNA damage leads to embryo development arrest. When sperm DNA is less damaged, embryo development is impaired, embryos show genomic instability, and if offspring are born, they may exhibit certain abnormalities such as altered adiposity and regulation of glucose in females [20,21].

Logically, the relevant experiments studying the relationship between sperm DNA damage and further embryo development were conducted mostly in rodents, where a relative high quality of cells (sperm, oocytes) can be obtained. Besides this, in vivo embryo techniques are almost perfected here. This is more complicated in domestic animals, where, for example, the oocytes are in vitro produced, ICSI needs to be perfected and if transferable embryos are produced they cannot be transferred into uteri in similarly high numbers as in rodents [22]. Moreover, the evaluation of DNA damage in intact spermatozoa is not very accurate because their chromatin is tightly packed with protamines. In our "Technology Report", we took the advantage that mammalian sperm heads decondense in foreign cytoplasm and form pronuclei, in which protamines are replaced with histones [17]. When bovine, ram, goat, horse and human sperm heads were injected into mouse ovulated oocytes, paternal pronuclei were always larger than maternal ones. Interestingly, in the pig the situation was quite the opposite, but even in this case, the level of sperm DNA damage could be more precisely evaluated than in intact spermatozoa. We observed a very interesting and unexpected situation when rooster spermatozoa were injected into mouse oocytes, when only a slight sperm head decondensation was observed. It has been supposed that a different P1/P2 ratio of different protamine compositions may be responsible for altered sperm head decondensation and subsequent formation of pronuclei [23]. These hypotheses are interesting and need to be confirmed by further studies.

Our approach can be used especially in those cases when the sperm number is quite low-oligospermic samples or in some specific cases, for example, when cadavers are recovered, as demonstrated already for somatic cell mammoth nuclei which were injected into mouse oocytes [24].

In general, in about two days, we can answer the question of whether the chosen sperm sample is suitable for further use or not or eventually if a given sperm storage method is convenient for sperm conservation or if it is necessary to modify it. Logically, it is necessary to find a threshold intensity of fluorescence, which will indicate that above that level the sperm DNA cannot be repaired by the oocyte, as it is well known that the oocyte contains DNA repair activities that are able to correct a low level (less than $8 \%$ ) of damaged sperm DNA [25].

\section{Conclusions}

In conclusion, in our contribution, we present a new approach for the evaluation of sperm DNA damage after ICSI into ovulated mouse oocytes. We are well aware that many factors may influence the quality of sperm, including the season, the method of sperm collection, freezing/thawing/lyophilization procedures, etc. The interspecific ICSI gives us 
an almost immediate possibility for rapid evaluation of the sperm samples and subsequent modifications of the above-mentioned approaches.

Author Contributions: Conceptualization of experiments, J.R., J.F.J., H.F., P.L., A.L., and M.B.; methodology, M.B., J.F.J., A.L., H.F., and J.R.; formal analysis, M.B. and J.F.J.; writing-original draft preparation, J.R., J.F.J. and M.B.; visualization, M.B.; project administration, J.R., J.F.J., and H.F. All authors have read and agreed to the published version of the manuscript.

Funding: This research was supported by the Ministry of Agriculture of the Czech Republic, institutional support MZE-RO0718 (V001). JR and JFJr are supported by NAZV 1910156. HF is supported by GACR 20-04465S. PL is supported by MIUR Demetra (2018-2022, CUP_C46C18000530001), and the European Union's Horizon 2020 Research and Innovation Program under the Marie SkłodowskaCurie grant agreements No. 734434.

Institutional Review Board Statement: The use of animals and the experimental design of this study were approved by the Institutional Animal Ethics Committee (approval number is 5/2018).

Conflicts of Interest: The authors declare that they have no conflict of interest.

\section{References}

1. Yanagimachi, R. Fertilization and development initiation in orthodox and unorthodox ways: From normal fertilization to cloning. Adv. Biophys. 2003, 37, 49-89. [CrossRef]

2. Yanagimachi, R. Intracytoplasmic injection of spermatozoa and spermatogenic cells: Its biology and applications in humans and animals. Reprod. Biomed. Online 2005, 10, 247-286. [CrossRef]

3. Wakayama, T.; Yanagimachi, R. Development of normal mice from oocytes injected with freeze-dried spermatozoa. Nat. Biotechnol. 1998, 16, 639-641. [CrossRef]

4. Ogonuki, N.; Mochida, K.; Miki, H.; Inoue, K.; Fray, M.; Iwaki, T.; Moriwaki, K.; Obata, Y.; Morozumi, K.; Yanagimachi, R.; et al. Spermatozoa and spermatids retriever from frozen reproductive organs or frozen whole bodies of male mice can produce normal offspring. Proc. Natl. Acad. Sci. USA 2006, 103, 13098-13103. [CrossRef] [PubMed]

5. Downey, A.M.; Robaire, B. Zygotic chromosomal structural aberrations after paternal drug treatment. Asian J. Androl. 2015, 17, 939-941.

6. Grenier, L.; Robaire, B.; Hales, B.F. Paternal cyclophosphamide exposure induces the formation of functional micronuclei during the first zygotic division. PLoS ONE 2011, 6, e27600. [CrossRef]

7. Gawecka, J.E.; Marth, J.; Ortega, M.; Yamauchi, Y.; Ward, M.A.; Ward, W.S. Mouse zygotes respond to severe DNA damage by delaying paternal DNA replication and embryonic development. PLoS ONE 2013, 8, e56385. [CrossRef] [PubMed]

8. Lewis, S.E.M.; Aitken, R.J. DNA damage to spermatozoa has impact on fertilization and pregnancy. Cell Tissue Res. 2005, 322, 33-41. [CrossRef]

9. Watanabe, H. Risk of chromosomal aberration in spermatozoa during intracytoplasmic sperm injection. J. Reprod. Dev. 2018, 64, 371-376. [CrossRef]

10. Conine, C.C.; Sun, F.; Song, L.; Rivera-Perez, J.A.; Rando, O. Sperm head preparation and genetic background affect caput sperm ICSI embryo viability: Cauda-enriched miRNAs only essential in specific conditions. Dev. Cell. 2020, 21, 677-678. [CrossRef]

11. Wang, Y.; Yamauchi, Y.; Wang, Z.; Zheng, H.; Yanagimachi, R.; Ward, M.A.; Yan, W. Both cauda and caput epididymal sperm are capable of supporting full-term development in FVB and CD-1 mice. Dev. Cell. 2020, 55, 675-676. [CrossRef]

12. Peris-Frau, P.; Soler, A.J.; Iniesta-Cuerda, M.; Martin-Maestro, A.; Sanchez-Ajofrin, I.; Medina-Chavez, O.G.; Fernandez-Santos, M.R.; Garcia-Alvarez, O.; Maroto-Morales, A.; Montoro, V.; et al. Sperm cryodamage in ruminants: Understanding the molecular changes induced by the cryopreservation process to optimize sperm quality. Int. J. Med. Sci. 2020, 21, 2781. [CrossRef]

13. Kumaresan, A.; Gupta, M.D.; Datta, T.K.; Morell, J.M. Sperm DNA integrity and male fertility in farm animals: A review. Front. Vet. Sci. 2020, 7, Art 321. [CrossRef]

14. Ladstatter, S.; Tachibana-Konwalski, K. A surveillance mechanism ensures repair of DNA lesions during zygotic reprogramming. Cell 2016, 167, 1774-1787. [CrossRef] [PubMed]

15. Kafer, G.R.; Li, X.; Horii, T.; Suetake, I.; Tajima, S.; Hatada, I.; Carlton, P.M. 5-hydroxymethylcytosine marks sites of DNA damage and promotes genome stability. Cell Rep. 2016, 14, 1-10. [CrossRef]

16. Derijck, A.; van der Heiden, G.; Giele, M.; Philippens, M.; de Boer, P. DNA double-strand break repair in parental chromatin of mouse zygotes, the first cell cycle as an origin of de novo mutation. Hum. Mol. Gen. 2008, 17, 1922-1937. [CrossRef] [PubMed]

17. Yoshida, N.; Perry, A.C.F. Piezo-actuated mouse intracytoplasmic sperm injection (ICSI). Nat. Protoc. 2007, 2, 296-304. [CrossRef]

18. Fulka, H.; Barnetova, I.; Mosko, T.; Fulka, J., Jr. Epigenetic analysis of human spermatozoa after their injection into ovulated mouse oocytes. Hum. Reprod. 2008, 23, 627-634. [CrossRef] [PubMed]

19. Shamsi, M.B.; Imam, S.N.; Dada, R. Sperm DNA integrity assays: Diagnostic and prognostic challenges and implications in management of infertility. J. Assist. Reprod. Genet. 2011, 28, 1073-1085. [CrossRef] [PubMed] 
20. Middelkamp, S.; van Tol, H.T.A.; Spierings, D.C.J.; Boymans, S.; Guryev, V.; Roelen, B.A.J.; Lansdorp, P.M.; Cuppen, E.; Kuik, E.W. Sperm DNA damage causes genomic instability in early embryonic development. Sci. Adv. 2020, 6, eaaz7602. [CrossRef]

21. Lane, M.; McPherson, N.O.; Fullston, T.; Spillane, M.; Sandeman, L.; Kang, W.X.; Zander-Fox, D.L. Oxidative stress in mouse sperm impairs embryo development, fetal growth and alters adiposity and glucose regulation in female offspring. PLoS ONE 2014, 9, e100832. [CrossRef] [PubMed]

22. Salamone, D.F.; Canel, N.G.; Rodriguez, M.B. Intracytoplasmic sperm injection in domestic and wild mammals. Reproduction 2017, 154, F111-F124. [CrossRef] [PubMed]

23. Maleszewski, M.; Kuretake, S.; Evenson, D.; Yanagimachi, H.; Bjordahl, J.; Yanagimachi, R. Behavior of transgenic mouse spermatoyoa with galline protamines. Biol. Reprod. 1998, 58, 8-14. [CrossRef] [PubMed]

24. Yamagata, K.; Nagai, K.; Miyamoto, H.; Anzai, M.; Kato, H.; Miyamoto, K.; Kurosaka, S.; Azuma, R.; Kolodeznikov, I.I.; Protopopov, A.V.; et al. Signs of biological activities of 28,000-year-old mammoth nuclei in mouse oocytes visualized by live-cell imaging. Sci. Rep. 2019, 9, 4050. [CrossRef]

25. Horta, F.; Catt, S.; Ramachandran, P.; Vollenhoven, B.; Temple-Smith, P. Female ageing affects the DNA repair capacity of oocytes in IVF using a controlled model of sperm DNA damage in mice. Hum. Reprod. 2020, 35, 529-544. [CrossRef] 